\title{
ContSOnto: A Formal Ontology for Continuity of Care
}

\author{
Subhashis DAS ${ }^{\mathrm{a}, 1}$ and Pamela HUSSEY ${ }^{\mathrm{b}}$ \\ ${ }^{a}$ CeIC, ADAPT, School of Computing \\ ${ }^{\mathrm{b}}$ CeIC, ADAPT, School of Nursing \& Human Sciences, \\ Dublin City University, Ireland
}

\begin{abstract}
The global pandemic over the past two years has reset societal agendas by identifying both strengths and weaknesses across all sectors. Focusing in particular on global health delivery, the ability of health care facilities to scale requirements and to meet service demands has detected the need for some national services and organisations to modernise their organisational processes and infrastructures. Core to requirements for modernisation is infrastructure to share information, specifically structural standardised approaches for both operational procedures and terminology services. Problems of data sharing (aka interoperability) is a main obstacle when patients are moving across healthcare facilities or travelling across border countries in cases where emergency treatment is needed. Experts in healthcare service delivery suggest that the best possible way to manage individual care is at home, using remote patient monitoring which ultimately reduces cost burden both for the citizen and service provider. Core to this practice will be advancing digitalisation of health care underpinned with safe integration and access to relevant and timely information. To tackle the data interoperability issue and provide a quality driven continuous flow of information from different health care information systems semantic terminology needs to be provided intact. In this paper we propose and present ContSonto a formal ontology for continuity of care based on ISO 13940:2015 ContSy and W3C Semantic Web Standards Language OWL (Web Ontology Language). ContSonto has several benefits including semantic interoperability, data harmonization and data linking. It can be use as a base model for data integration for different healthcare information models to generate knowledge graph to support shared care and decision making.
\end{abstract}

Keywords. EHR, Interoperability, Semantic, Ontology, OWL

\section{Introduction}

The global crisis caused due to the ongoing pandemic, has largely altered the functionality of various service industries including healthcare sectors. Healthcare sector issues include but are not restricted to a lack of conformance with standards use or vendor lock in due to use of proprietary Electronic Healthcare Records (EHRs) software and systems which are unable to exchange data within and across government organizations. However, few Artificial Intelligence (AI) companies are promising that AI based solution will solve this issue by providing intelligent allocation of resources among care facilities such as beds, doctors, and patients. Recently there is a proliferation of white

${ }^{1}$ Corresponding Author. Subhashis Das, CeIC, ADAPT, School of Computing, Dublin City University (DCU), Dublin 9, Ireland; E-mail: subhashis.das@dcu.ie. 
papers reports and publications reflecting the buzz around Artificial Intelligence (AI) based healthcare $[1,8]$. AI however can only be realised with standard data exchange which is machine understandable at the same time capable of aggregate data from various sources [15].

To tackle this data exchange issue across health systems in this paper we propose a formal ontology of Continuity of care (ContSOnto). ContSOnto as an emerging research area consisting of the extension of healthcare ontology to the continuity of care domain. This field is positioned at the confluence of health informatics, nursing informatics, process modeling, and artificial intelligence. Gulliford et al. (2006) [7] describe "Continuity of care as a process which is concerned with the quality of care over time". There are two aspects to this perspective. One is based on the patient's experience of a 'continuous caring relationship' with the healthcare professional. Another one is based on a system of care where seamless service is needed to provide care through integration, coordination and interoperable information systems. WHO (2018) [14] defines Continuity of care as reflects the extent to which a series of discrete health care events is experienced by people as coherent and interconnected over time and consistent with their health needs and preferences. To develop our ContSOnto model we engaged with healthcare professionals as well as standards bodies who originally were involved in development work of ISO 13940:2015 ContSys. Earlier Horizon 2020 project Hospital at Home (H@H) project [12] proposed a conceptual model of social care to be included in the care system but model does not provide an outline for any real implementation after five years. Although those $\mathrm{H} @ \mathrm{H}$ conceptual model is not based on Resource Description Framework (RDF) it therefore hinder the main objective of data exchange with other healthcare systems. ContSOnto model align with the European ISA recommendation on new European Interoperability Framework (EIF) [3] and describes how ContSOnto is conforming with EIF level as depicted in Figure1.

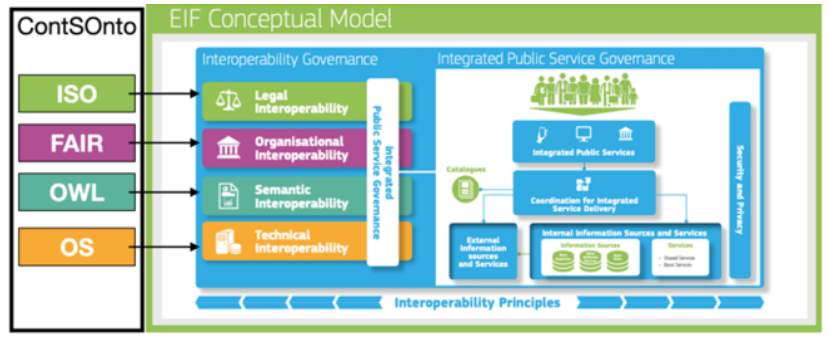

Figure 1:ContSOnto Alignment with New EIF

The International Standard Organization (ISO) provides a legal framework for intergovernmental interoperability. The acronym FAIR equates to data that is Findability, Accessibility, Interoperability, and Reusable. The FAIR principle allowed ContSOnto organizational Interoperability, as ContSOnto model is based on Web Ontology Language (OWL) which enables the most needed se- mantic interoperability ecosystem [2]. In addition, the process of ContSOnto is focused on developing using open source (OS) software and Open protocol (Open API), thus ContSOnto is neutral and does not rely on any proprietary software. This paper is structured as follows: In Section 2, we describe the overall methodology, in Section 3 results and implementation, and we conclude in Section 4 with a discussion on future work. 


\section{Methodology}

ContSOnto development methodology is based on two main features. One is requirements analysis to verify what is needed to have for a care model so that it fulfills mod- ern's days software (i.e. app) needs described in Section 2.1. Other aspect is to develop a model based on ontological decisions as stated in OntoClean methodology [6] described in Section 2.2.

\subsection{Requirements}

How the ContSys standards are approached, designed, constrained, or extended is based on a formal logical model. The ContSys ontology model therefore needs to be mapped explicitly to Resource Description Framework (RDF) formalism as per W3C Semantic Web Standards. Without such a model to operate from, ContSys Ontology will lack the semantic and structural consistency required to make ContSys computable and generate knowledge graphs. Priorities are indicated using MoSCoW terms (MUST, SHOULD, COULD, WON'T). 1) ContSys Ontology Mappings (MUST): We shall define lossless bi-directional transformations from ContSys UML instances to OWL/RDFS ontology representations and vice versa. 2) Complete ContSys Coverage (MUST): The RDF representation of ContSys Unified Modeling Language (UML) element instance data shall be capable of expressing all legal ContSys instances that make use of any valid ContSys sub-set, including extensions. An RDF instance data representation that is limited to only a subset of possible ContSys instances is not acceptable. 3) Monotonic with Modifier Extensions (MUST): ContSys RDF data with modifier extensions shall be "consistent" for RDF reasoning, i.e., the semantics of the RDF must be monotonic even in the presence of modifier extensions. 4) Vocabulary Bindings (MUST): The ContSys ontology shall support vocabulary bindings to code, Coding and Codeable Concept - including dealing with extensible value sets and multi-code system value sets. (SHOULD) The ContSys vocabulary representation should be able to leverage existing semantic web terminology representations (e.g., SNOMED-CT). 5) Enforce Constraints (SHOULD): The ContSys ontology should enforce constraints that are representable in OWL/RDF whenever possible, e.g., schema constraints, regular expressions, etc. 6) Annotation In- formation (SHOULD): In the RDFS/OWL Ontology representation, should expose at least minimal annotation information for display in an ontology editor for use by humans. 7) Top-level alignment (SHOULD): ContSys Ontology should be aligned with one top-level ontology. 8) RDF Quality (MUST): Transformations into RDF must meet software quality checks including ontological closure. The RDF instance which is transformed from contsys UML must be capable of being opened without further modification by widely available tools including Protégé.

\subsection{Formal Ontology}

Gruber (1993) [4] defined ontology as a "formal, explicit specification of a shared conceptualization". Ontology provides a shared vocabulary, which can be used to model a domain of discourse that is, the type of objects, and/or concepts that exist, and their properties and relations. As per Guarino (1998) [5] Ontology is "a set of logical axioms designed to account for the intended meaning of a vocabulary". In this definition, Guarino emphasized the role of logic as a way of representing an ontology. Need for 
building a formal ontology for contsys highlighted by Martınez-Costa et al. (2015) [11]. We believe that ontology has an important role to play in the general task of managing diverse information.

The purpose of defining a Resource Description Framework (RDF) representation of ContSOnto is not only to enable ContSOnto to be exchanged in an RDF format such as Turtle, JSON-LD but also to ground the semantics of ContSOnto data in RDF, for use with ontologies and other RDF data. Since the ContSOnto data model is losslessly assembled, any component of the data model can be used in conjunction with RDF. The semantics are well kept regardless of source format. We choose Web Ontology Language (OWL) for formalization language. It is built upon the World Wide Web Consortium's (W3C) XML standard for objects called the RDF. OWL provides the benefit of reasoning using Description Logic (DL). More precisely we choose OWL 2 for modeling. It has five main advantages than the previous version such as property chains; richer data types, data ranges; qualified cardinality restrictions; asymmetric, reflexive, and disjoint properties; and enhanced annotation capabilities [9].

\subsection{Ontology Alignment}

\begin{tabular}{|l|l|}
\hline Class hierarchy: owl:Thing & Inferred \\
\hline owent \\
adverseEvent \\
appointment \\
automatedHealthcare \\
contact \\
demandForCare \\
dissent \\
health_thread \\
healthcare \\
healthcare_activity_element \\
healthcare_administration \\
healthcareActivity \\
healthcareCommitment \\
healthRelatedPeriod \\
service \\
mentalObject \\
organization \\
physicalObject \\
location \\
medicalDevice \\
medium \\
person \\
product \\
resource \\
healthcare_funds \\
healthcare_resource \\
role \\
healthcare_actor \\
healthcare_personnel \\
healthcare_provider \\
healthcare_third_party \\
subjectofCare \\
parganizationRole \\
personRole \\
stative \\
process \\
state
\end{tabular}

Top-level ontologies provide domain-independent conceptualization, relations, and axioms (e.g., categories like Event, Mental Object, Quality, etc.) in order to standardize upper-level of a model thus enable linking con ontology with other freely available ontology such Link data vocabulary $(\mathrm{LOV})^{2}$ and Biomedical Ontology by $\mathrm{NCBO}^{3}$. In ContSOnto we use the top-level ontology Descriptive Ontology for Linguistic and Cognitive Engineering (DOLCE) [5] as a middle-out solution between degree of formalization and complexity, contributing to an effective practical solution. In spite of the benefit of top-level ontologies, their alignment and use is not trivial and requires some expert effort. The EU project Advancing Clinico-Genomic Trials (ACGT) [13] as well as other healthcare projects emphasis on need and benefit from top-level alignment. Figure 2 depicted Class hierarchy of ContSOnto ontology. And Figure 3 showcase partial view of ContSOnto class visualization using WebProtégé tool and upper part of the figure in green such as mentalObject, stative, event are DOLCE classes and other are domain specific class taken from ISO 13940:2015 ContSys.

Figure 2. Class Hierarchy

\footnotetext{
2 https://lov.linkeddata.es/dataset/lov/

3 https://bioportal.bioontology.org/ontologies
} 


\section{Results and Implementation}

The resulting formal ontology is available online on National Center for Biomedical Ontology (NCBO) Bioportal ContSOnto and full Ontology documentation on GitHub.

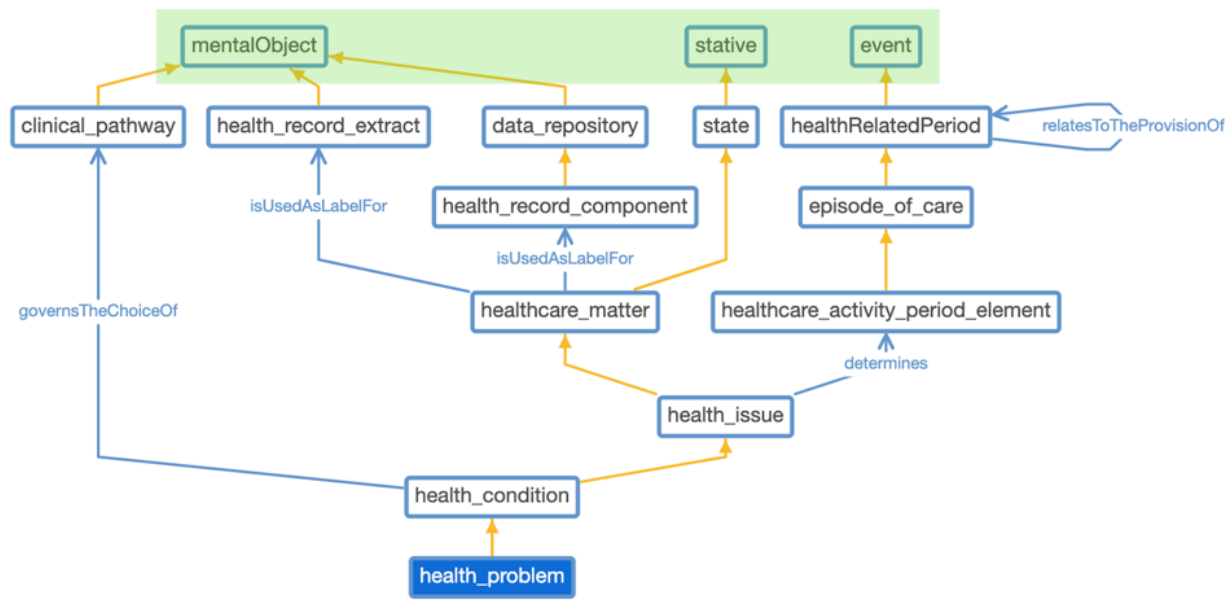

Figure 3. ContSOnto Alignment with DOLCE Top-level ontology (DOLCE classes are in grey)

repository with permanent URI http://purl.org/net/for-coc. In its current version, it is based on ISO 13940:2015 ContSys. It consists of 21888 triples. A total of 153 OWL Classes and 144 OWL Properties have been defined. ContSOnto has total 961 axiom with 415 logical axioms and 305 declarative axioms. Expressiveness of ContSOnto model is $A L C H Q(D)$ as per description logic (DL) scale.

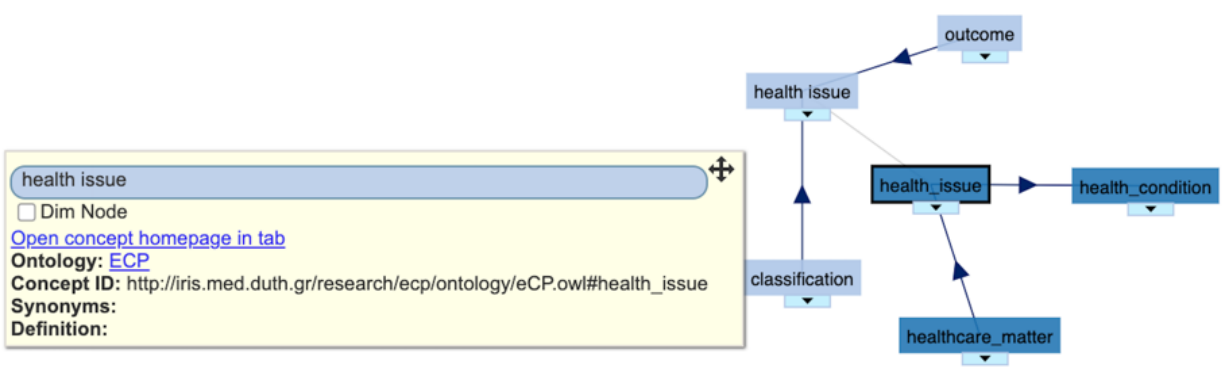

Figure 4. Neighborhood relation with ECP ontology using Bioportal web service

\section{Discussion}

The pandemic has presented many challenges globally, and health researchers, policy analysts and decision makers are reporting worrying results on predictive models for 2020-2021. Whereas connection among different healthcare settings still has a long path to progress, In this direction, ContSOnto can be seen as a base model which will provide scope for wider collaboration. For example SNOMED International will publish ICNP Reference Sets and an associated ontology in September 2021, as the CeIC is an ICNP 
R\&D Center ${ }^{4}$ future progression of nursing sensitive data to advance patient centered integrated care models is under consideration. Initial research on development of Nursing Knowledge Graph (NKG) and our progress in this domain is published and available to view from Journal of Nursing Scholarship [10]. The benefits of using NCBO Bioportal is that we can leverage its online annotation and semantic matching facilities to discover other related models available on the Bioportal using Neighborhood matching. Figure 4 above provides one such example, which showcase associated between ContSOnto's Health issue (node in dark blue) with ECP ontology (node in light blue).

\section{Acknowledgement}

This research has received funding from the European Union's Horizon 2020 re- search and innovation programme under the ELITE-S Marie Skłodowska-Curie grant agreement No. 801522, by Science Foundation Ireland and co-funded by the European Regional Development Fund through the ADAPT Centre for Digital Content Technology grant number 13/RC/2106 P2 and DAVRA Networks.

\section{References}

[1] Bhattacharya S, Pradhan KB, Bashar MA, Tripathi S, et al. Artificial intelligence enabled healthcare: A hype, hope or harm. Journal of family medicine and primary care. 2019 Nov;8 (11): 3461.

[2] Blobel B. Interoperable EHR systems-challenges, standards and solutions. European Journal for Biomedical Informatics. 2018; 14(2): 10-9.

[3] European Commission. New european interoperability framework, 2017.

[4] Gruber TR. A translation approach to portable ontology specifications. Knowledge acquisition. 1993 Jun $1 ; 5(2): 199-220$.

[5] Guarino N, editor. Formal ontology in information systems: Proceedings of the first international conference (FOIS'98), June 6-8, Trento, Italy. IOS press; 1998.

[6] Guarino N, Welty C. Evaluating ontological decisions with OntoClean. Communications of the ACM. 2002 Feb 1;45(2):61-5.

[7] Gulliford M, Naithani S, Morgan M. What is' continuity of care'?. Journal of health services research \& policy. 2006 Oct $1 ; 11(4): 248-50$.

[8] Han C, Rundo L, Murao K, Nemoto T, Nakayama H. Bridging the gap between AI and healthcare sides: towards developing clinically relevant AI-powered diagnosis systems. InIFIP International Conference on Artificial Intelligence Applications and Innovations 2020 Jun 5 (pp. 320-333). Springer, Cham.

[9] Hitzler P, Krötzsch M, Parsia B, Patel-Schneider PF, Rudolph S. OWL 2 web ontology language primer. W3C recommendation. 2009 Oct 27; 27(1): 123.

[10] Hussey P, Das S, Farrell S, Ledger L, Spencer A. A Knowledge Graph to Understand Nursing Big Data: Case Example for Guidance. Journal of Nursing Scholarship. 2021 May; 53(3): 323-32.

[11] Martínez-Costa C, Kay S, Oughtibridge N, Schulz S. ContSys under Ontological Scrutiny. InDigital Healthcare Empowering Europeans 2015 (pp. 999-999). IOS Press.

[12] Pecoraro F, Luzi D, Ricci FL. An integrated model to capture the provision of health and social care services based on the ContSys and FHIR standards. EJBI. 2017; 13(1): 17-26.

[13] Stenzhorn H, Schulz S, Boeker M, Smith B. Adapting clinical ontologies in real-world environments. Journal of Universal Computer Science. 2008; 14(22).

[14] WHO. Continuity and coordination of care, 2018.

[15] WHO. Ethics and governance of artificial intelligence for health who guidance, 2021.

\footnotetext{
${ }^{4}$ https://www.icn.ch/what-we-doprojectsehealth-icnpabout-icnpicn-accredited-centres-icnpr-researchdevelopment/icn
} 